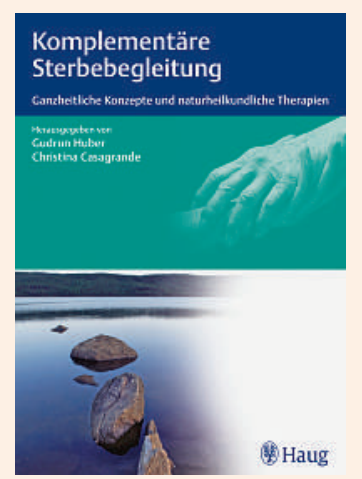

Gudrun Huber, Christina Casagrande (Hrsg.) Komplementäre Sterbebegleitung

Ganzheitliche Konzepte und naturheilkundliche Therapien

Stuttgart: Haug; 2011

230 Seiten, $70 \mathrm{CHF}$ ISBN 978-3-8304-7347-3

\title{
Komplementäre Therapien für Sterbende
}

Diese Neuerscheinung richtet sich an alle stationär oder ambulant in der Palliative Care Tätigen, an Fachleute wie Ärzte, Therapeutinnen oder Pflegende, aber auch an begleitende Angehörige und Freunde sterbender Patienten. Das Buch ist in erster Linie ein Praxisleitfaden. Nach einer kurzen theoretischen Einführung im ersten Teil, sowohl in die Kulturgeschichte unseres Umgangs mit dem Tod (spannend!) wie in die wissenschaftlichen Grundlagen des Sterbeprozesses, die Bedürfnisse Sterbender und ihrer Angehörigen, sowie die Rolle des Therapeuten und Begleiters, ist der zweite, weitaus umfangreichere Teil des Buchs ganz der Praxis gewidmet. Zuerst werden die bewährten komplementären Therapieverfahren kurz vorgestellt, von A wie Aromatherapie bis S wie Spagyrik. Danach folgt der Hauptteil, in dem indikationsbezogen und rezeptbuchartig mögliche komplementärmedizinische Massnahmen aufgeführt werden. Von Dekubitus über Dyspnoe und Schlaflosigkeit bis hin zu Dysurie oder Schwitzen, aber auch bei verschiedenen psychischen Symptomen: bei jedem Problem sind mögliche Anwendungen aus Anthroposophischer Therapie, Homöopathie, Spagyrik, Bachblütentherapie, Phyto- oder Aromatherapie, Entspannungsverfahren, Yoga oder Atemtherapie aufgeführt, mit knappen, aber klaren Angaben zu Indikation, Dosierung, Dauer usw. Und dies stets unter Berücksichtigung des Möglichen, will heissen auch der Grenzen, die Nicht-Fachleuten der vorgestellten komplementären Therapiesysteme naturgemäss gegeben sind. Wo diese Beschränkung bei Methoden wie Phyto- oder Aromatherapie wenig ins Gewicht fällt, führt dies bei komplexen Systemen wie der klassischen Homöopathie allerdings teilweise zu einer allzu grossen Vereinfachung und zur Einschränkung auf bloss zwei, drei Mittel pro Indikation, hier wäre meines Erachtens etwas mehr Breite möglich gewesen.

Am Ende des Buchs finden sich noch wichtige Kapitel über die spirituelle Begleitung Sterbender sowie über die Trauerbegleitung der Angehörigen und deren mögliche komplementärmedizinische Begleitung. Aus hiesiger Sicht ist es etwas schade, dass im Anhang der Adressteil vor allem solche aus Deutschland enthält und entsprechende aus der Schweiz (oder Österreich) weitgehend fehlen, hier sollte bei weiteren Auflagen unbedingt nachgebessert werden.

Von den erwähnten kleinen Mängeln abgesehen, ist das Buch aber eine wahre Fundgrube guter, praktischer Ideen und Anleitungen und wird wohl speziell bei Pflegenden - dem Komplementärmedizinischen in der Regel offener gegenüberstehend als wir Ärzte - in Palliative-Care-Einrichtungen hoffentlich seine Verbreitung finden.

Dr. med. Alexander Erlach, Winterthur 